\title{
ON THE UNIQUENESS OF D-VERTEX MAGIC CONSTANT
}

\author{
S. Arumugam ${ }^{1}$, N. Kamatchi ${ }^{2}$ \\ National Centre for Advanced Research \\ in Discrete Mathematics (n-CARDMATH) \\ Kalasalingam University \\ Anand Nagar, Krishnankoil-626 126, Tamil Nadu, India \\ e-mail: s.arumugam.klu@gmail.com \\ n_kamatchi@yahoo.com \\ AND \\ G.R. VIJAYAKUMAR \\ School of Mathematics \\ Tata Institute of Fundamental Research \\ Homi Bhabha Road, Colaba, Mumbai 400 005, India \\ e-mail: vijay@math.tifr.res.in
}

\begin{abstract}
Let $G=(V, E)$ be a graph of order $n$ and let $D \subseteq\{0,1,2,3, \ldots\}$. For $v \in V$, let $N_{D}(v)=\{u \in V: d(u, v) \in D\}$. The graph $G$ is said to be $D$-vertex magic if there exists a bijection $f: V(G) \rightarrow\{1,2, \ldots, n\}$ such that for all $v \in V, \sum_{u \in N_{D}(v)} f(u)$ is a constant, called $D$-vertex magic constant. O'Neal and Slater have proved the uniqueness of the $D$-vertex magic constant by showing that it can be determined by the $D$-neighborhood fractional domination number of the graph. In this paper we give a simple and elegant proof of this result. Using this result, we investigate the existence of distance magic labelings of complete $r$-partite graphs where $r \geq 4$.
\end{abstract}

Keywords: distance magic graph, $D$-vertex magic graph, magic constant, dominating function, fractional domination number.

2010 Mathematics Subject Classification: 05C78, 05 C69.

\footnotetext{
${ }^{1}$ Also at School of Electrical Engineering and Computer Science, The University of Newcastle, NSW 2308, Australia; Department of Computer Science, Liverpool Hope University, Liverpool, UK; Department of Computer Science, Ball State University, USA.

${ }^{2}$ The first and second authors are thankful to the Department of Science and Technology, New Delhi, Government of India for its support through $n$-CARDMATH Project SR/S4/MS:427/07.
} 


\section{REFERENCES}

[1] S. Arumugam, D. Fronček and N. Kamatchi, Distance magic graphs-A survey, J. Indones. Math. Soc., Special Edition (2011) 11-26.

[2] S. Beena, On $\Sigma$ and $\Sigma^{\prime}$ labelled graphs, Discrete Math. 309 (2009) 1783-1787. doi:10.1016/j.disc.2008.02.038

[3] G. Chartrand and L. Lesniak, Graphs \& Digraphs, $4^{\text {th }}$ Edition (Chapman and Hall, CRC, 2005).

[4] D. Grinstead and P.J. Slater, Fractional domination and fractional packings in graphs, Congr. Numer. 71 (1990) 153-172.

[5] T.W. Haynes, S.T. Hedetniemi and P.J. Slater, Domination in Graphs, Advanced Topics (Marcel Dekker, Inc., 1998).

[6] M. Miller, C. Rodger and R. Simanjuntak, Distance magic labelings of graphs, Australas. J. Combin. 28 (2003) 305-315.

[7] A. O'Neal and P.J. Slater, An introduction to distance D magic graphs, J. Indones. Math. Soc., Special Edition (2011) 91-107.

[8] A. O'Neal and P.J. Slater, Uniqueness of vertex magic constants, SIAM J. Discrete Math. 27 (2013) 708-716.

doi:10.1137/110834421

[9] K.A. Sugeng, D. Fronček, M. Miller, J. Ryan and J. Walker, On distance magic labeling of graphs, J. Combin. Math. Combin. Comput. 71 (2009) 39-48.

[10] V. Vilfred, $\Sigma$-labelled graph and circulant graphs, Ph.D. Thesis, University of Kerala, Trivandrum, India, 1994.

Received 21 September 2012

Revised 14 March 2013

Accepted 14 March 2013 